\title{
Re-deployment of a dislodged lumen-apposing metal stent (LAMS): avoiding the sacrificial LAMS
}

Lumen-apposing metal stents, or LAMS, have been increasingly used for endoscopic drainage of walled-off pancreatic necrosis (WON). However, these can become dislodged during endoscopic necrosectomy and are expensive to replace. This video highlights a technique to reuse a LAMS after it has become dislodged ( $\triangleright$ Video 1 ).

A 63-year-old man with necrotizing pancreatitis underwent endoscopic ultrasound-guided drainage with placement of a $15 \times 10 \mathrm{~mm}$ LAMS (AXIOS; Boston Scientific, Marlborough, Massachusetts, USA) for an $18-\mathrm{cm}$ area of WON. The patient returned for endoscopic necrosectomy 4 weeks after initial placement. A large amount of necrosis was noted to be obstructing the LAMS ( $\triangleright$ Fig. 1). During initial debridement, the stent became dislodged and could not be replaced, and was therefore removed from

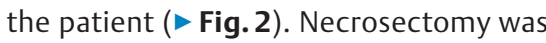
performed using a snare and rat tooth forceps. Although the majority of the necrosis was removed, a small amount of thick adherent material could not be extracted. Therefore the decision was made to replace the LAMS.

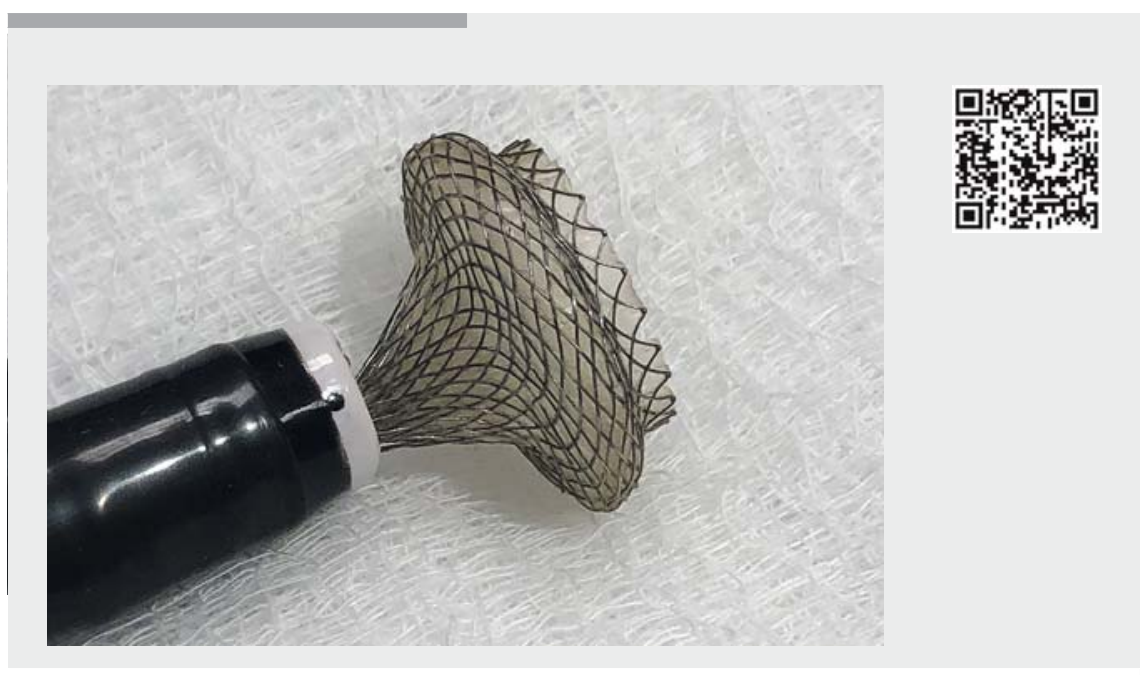

$\checkmark$ Video 1 Re-deployment of a lumen-apposing metal stent after dislodgment during endoscopic necrosectomy.
The LAMS was thoroughly washed in sterile water. By squeezing and elongating the LAMS, it was back loaded into the channel of the therapeutic upper endoscope. A short portion of the distal aspect of the LAMS was intentionally left outside the channel in case it needed to be recovered ( $\triangleright$ Fig.3). A 5-10 Fr Soehendra biliary dilation catheter (Cook Medical, Winston-Salem, North Carolina, USA) was modified by cutting off the graded portion so that the distal tip was $10 \mathrm{Fr}$ in diameter, and the modified catheter was advanced down the channel to the level of the stent. The endoscope was then advanced into the cavity, and under endoscopic and fluoroscopic guidance the distal flange was deployed by advancing the dilation catheter. The endoscope was then withdrawn across the cystgastrostomy tract, and when in the gastric lumen, the proximal flange was deployed (\$ Fig.4). The endoscope was then advanced through the stent to confirm successful deployment.

Endoscopy_UCTN_Code_CPL_1AK_2AG

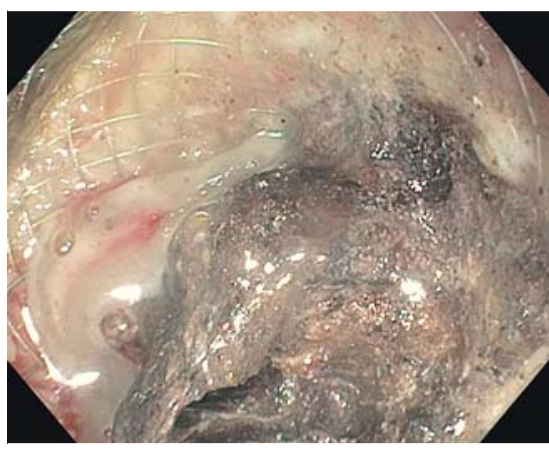

- Fig. 1 Necrotic material occluding the lumen-apposing metal stent.

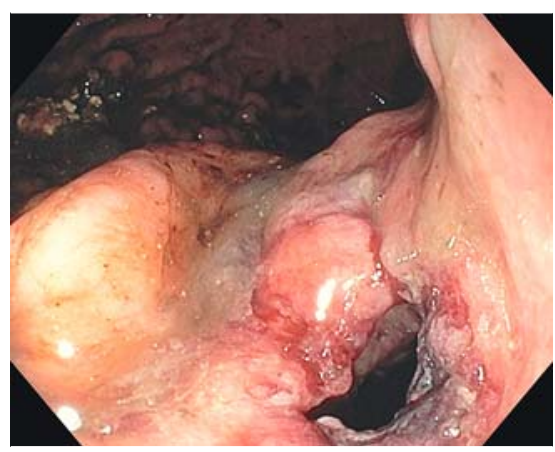

- Fig. 2 Cystgastrostomy tract after removal of the lumen-apposing metal stent.

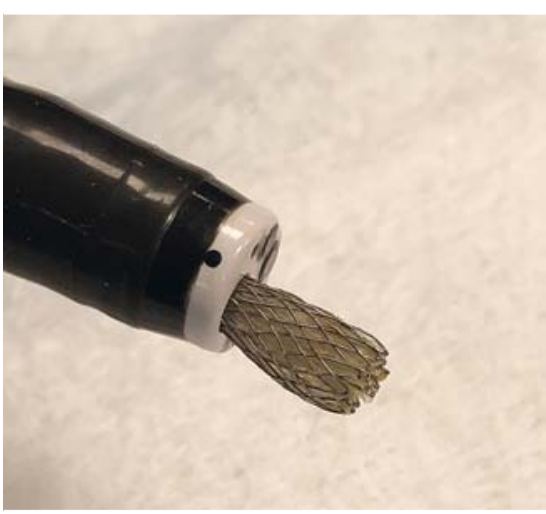

- Fig. 3 Fully collapsed lumen-apposing metal stent loaded in the channel of the therapeutic upper endoscope. 


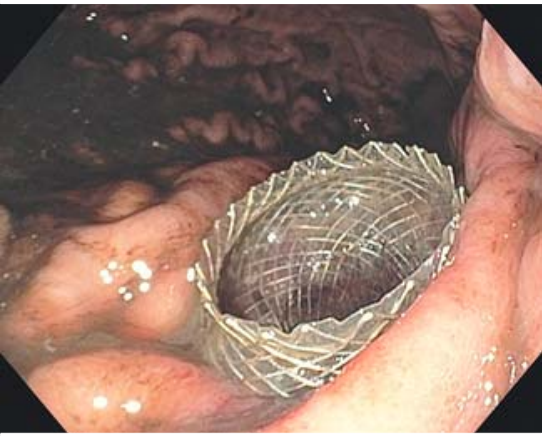

- Fig. 4 Successful re-deployment of the lumen-apposing metal stent through the cystgastrostomy tract.

Competing interests

Vinay Chandrasekhara has undertaken consultancy work for Boston Scientific.
The authors

Vinay Chandrasekhara, Mark Topazian, Barham K. Abu Dayyeh, Michael J. Levy

Gastroenterology and Hepatology, Mayo Clinic, Rochester, United States

Corresponding author

\section{Vinay Chandrasekhara, MD}

Gastroenterology and Hepatology, Mayo Clinic, 200 First St SW, Rochester, MN 55905 United States

Fax: +1-507-538-5820

Chandrasekhara.vinay@mayo.edu

\section{Bibliography}

DOI https://doi.org/10.1055/a-0624-1576

Published online: 19.6.2018

Endoscopy 2018; 50: E233-E234

(c) Georg Thieme Verlag KG

Stuttgart · New York

ISSN 0013-726X

\section{ENDOSCOPY E-VIDEOS \\ https://eref.thieme.de/e-videos}

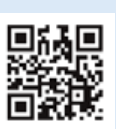

Endoscopy E-Videos is a free access online section, reporting on interesting cases and new techniques in gastroenterological endoscopy. All papers include a high quality video and all contributions are freely accessible online.

This section has its own submission website at https://mc.manuscriptcentral.com/e-videos 